\title{
Quorum Sensing Inhibitory Activities of Oridonin in Pseudomonas Aeruginosa
}

\author{
Jing Huang ${ }^{1}$, Haoming $\mathrm{Li}^{1 *}$ \\ ${ }^{1}$ School of Biosciences and Biopharmaceutics, Guangdong Pharmaceutical University, Guangzhou 510006, China
}

\begin{abstract}
Pseudomonas aeruginosa is an opportunistic pathogen, and it can produce cell-associated and extracellular virulence factors. Several of these virulence factors have been demonstrated to be regulated by quorum sensing (QS). Disabling QS system with anti-infective agent is considered as a potential strategy to prevent bacterial infection. Rabdosia rubescens has been used as antibacterial agents for many centuries in China. In this study, Oridonin, the major active components of Rabdosia rubescens, was tested for QS inhibition in Pseudomonas aeruginosa. QS inhibitory activity is demonstrated by reduction in pyocyanin (58.4\%), rhamnolipids (64.3\%), elastase (58.6\%), and protease (49.1\%) in Pseudomonas aeruginosa PAO1 at $125 \mu \mathrm{g} / \mathrm{ml}$ (MIC) concentration. Biofilm formation by Pseudomonas aeruginosa PAO1 was reduced considerably (40.3$57.7 \%$ ) over control. These findings suggest that Oridonin might be a potent Quorum Sensing Inhibitor (QSI) and anti-biofilm agent in the treatment of Pseudomonas aeruginosa infections.
\end{abstract}

\section{Introduction}

Bacterial infection has been a major problem on the medical treatment. With the introduction of natural antibiotics such as penicillin, antibiotic derivatives and synthetic antibiotics, they have opened a new era in the treatment of bacterial infections[1]. However, with the widely abuse of antibiotics, drug resistance is becoming a severe problem, at the same time, the emergence of multiresistant bacteria and mutation time of drug resistance also become more and more short[2], so it is urgent to find new targets of antibacterial agents and pathways to treat diseases.

Quorum Sensing (QS) found in Gram-negative and Gram-positive bacteria. Bacteria could produce some signaling molecules, called autoinducers (AI), that can coordinate the physiological activities as well as the expression of virulence factors in the pathogenesis[3]. The attenuation of virulence factors and pathogenicity of bacteria through interfering QS is a possible alternative to killing or inhibiting growth of pathogenic bacteria. Many researchers have indicated that phytochemicals inhibit virulence factor production and biofilm formation by interfering auto-induce signaling molecules in quorum sensing system.

P. aeruginosa is an opportunistic pathogenic bacterium, which can be easily detected in the skin and lung infections. QS plays a significant role in the regulation of $\mathrm{P}$. aeruginosa virulence expression such as biofilm,

\footnotetext{
*Corresponding author: lihaoming@aliyun.com
}

rhamnolipid, pyocyanin, elastase, and protease[4]. Bacterial biofilm development depends on release of extracellular polymeric compounds. Rhamnolipid promotes biofilm formation and diffusion, Elastases and proteases play key roles in early invasion and disintegration of host cells $[5,6]$.

Rabdosia rubescens, a Traditional Chinese Medicine (TCM), has been used in reducing fever, antidiarrhea. It has a significant effect on acute laryngitis and suppurative tonsillitis, and also has a good effect on chronic bronchitis and chronic pharyngitis[7]. The main active component of Rabdosia rubescens is oridonin, a natural diterpene carvacene organic compound. In this work, we aim to study the potential of oridonin as quorum sensing inhibitor.

\section{Methods}

\subsection{Bacterial strains and culture conditions}

$P$. aeruginosa PAO1 was routinely cultured in LuriaBertani (LB) broth at $37^{\circ} \mathrm{C}$ with shaking. Bacterial culture with an optical density of 0.5 at $600 \mathrm{~nm}$ was used for all studies.

\subsection{MIC Determination}

Minimum inhibitory concentration (MIC) was determined by the broth micro-dilution method (Clinical and Laboratory Standards Institute, CLSI 2015) by using 96- 
well microtiter plates. Serial two-fold dilutions method was used in this assay, $100 \mu 1$ of Oridonin(Sigma) solution and $100 \mu \mathrm{l}$ of LB broth were placed into the first well of a microtiter plate and mixed evenly, extra $10 \mu \mathrm{l}$ PAO1 cultures was added in every well and incubated at $37^{\circ} \mathrm{C}$. The minimum concentration with no visible bacterial growth was defined as MIC.

\subsection{Pyocyanin Assay}

Oridonin solution was mixed well with overnight grown PAO1 cultures and incubated at $37^{\circ} \mathrm{C}$ for $24 \mathrm{~h}$. Pyocyanin was extracted with chloroform and vortexed vigorously. Then, the mixed cultures were centrifuged at 10000rpm for $10 \mathrm{~min}$. The chloroform layer was re-extracted with $0.2 \mathrm{~mol} / \mathrm{l} \mathrm{HCl}$. After the same centrifugation, $200 \mu \mathrm{l}$ of the $\mathrm{HCl}$ layer solution were transferred to 96-well plates and the absorbance was read at $570 \mathrm{~nm}[8]$.

\subsection{Rhamnolipids assay}

Overnight grown PAO1 culture was adjusted to OD600 0.5 by PPGAS broth $(20 \mathrm{mM} \mathrm{NH} 4 \mathrm{Cl}, 20 \mathrm{mM} \mathrm{KCl}, 1.6 \mathrm{mM}$ $\mathrm{MgSO} 4,0.5 \%$ Glucose, $1.0 \%$ Peptone, $120 \mathrm{mM}$ Tris- $\mathrm{HCl}$ every $1000 \mathrm{~mL}, \mathrm{pH}$ 7.2). Oridonin solution was mixed with PAO1 cultures to make the test concentrations, incubated at $37^{\circ} \mathrm{C}$ for $24 \mathrm{~h}$. After centrifugation $(8000 \mathrm{rpm}$ for $10 \mathrm{~min}$ at room temperature), the supernatant was adjusted to $\mathrm{pH} 2.0$, and extracted with ethyl acetate twice, evaporate to dryness in a drying chamber. The sediment was re-dissolve in $\mathrm{ddH} 2 \mathrm{O}$. The resulting solution was mixed completely with sulfuric acid $(800 \mu \mathrm{l}, 60 \%$ (v/v)) and orcinol $(1.7 \%, 100 \mu 1) .200 \mu 1$ of solution were transferred to 96-well plates and the absorbance was read at $421 \mathrm{~nm}[9]$.

\subsection{Protease Activity Assay}

Overnight grown PAO1 culture was adjusted to OD600 0.5 by LB broth. Oridonin solution was added to PAO1 cultures to make the test concentrations, incubated at $37^{\circ} \mathrm{C}$ for $24 \mathrm{~h}$. PAO1 cultures were centrifuged (10000 rpm for $10 \mathrm{~min}$ at room temperature) and then filtered through 0.22 $\mu \mathrm{m}$ filter membrane. Culture supernatant $(150 \mu \mathrm{l})$ were added to $250 \mu \mathrm{l}$ of $2 \%(\mathrm{w} / \mathrm{v})$ azocasein-Tris- $\mathrm{HCl}$ solution, stored at $4{ }^{\circ} \mathrm{C}$ for 4 hours, Trichloroacetic acid $(10 \%, 1.2 \mathrm{ml})$ were added to terminate the reaction and then centrifuged at $10000 \mathrm{rpm}$ for $10 \mathrm{~min}$. Finally, $200 \mu \mathrm{L}$ of solution were transferred to 96-well plates and the absorbance was read at $440 \mathrm{~nm}[10]$.

\subsection{Elastase Assay}

Oridonin was added to PAO1 cultures to make the test concentrations, incubated at $37^{\circ} \mathrm{C}$ for $24 \mathrm{~h}$. PAO1 cultures were centrifuged $(6000 \mathrm{rpm}$ for $10 \mathrm{~min}$ at room temperature) and then filtered through $0.22 \mu \mathrm{m}$ filter membrane. Culture supernatant $(200 \mu \mathrm{l})$ was mixed with Congo red elastin solution $(800 \mu 1,2 \%, \mathrm{pH} 7.5)$, incubated at $37^{\circ} \mathrm{C}$ for $4 \mathrm{~h}$, centrifuged at $10000 \mathrm{rpm}$ for $10 \mathrm{~min} .200$ $\mu \mathrm{L}$ of solution were transferred to $96-$ well plates and the absorbance was read at $495 \mathrm{~nm}[11]$.

\subsection{The semiquantitative of Biofilm}

Sterilized coverslip as adhesive carrier $(2 \mathrm{~cm} \times 2 \mathrm{~cm})$ was placed in a sterilized 6-well microtiter plate. Oridonin solution $(2 \mathrm{ml})$ and PAO1 culture $(20 \mu \mathrm{l})$ were added in each well, incubated at $37^{\circ} \mathrm{C}$ for 7 days. Within the seven days, bacteria that did not form biofilm was removed by sterilized PBS (pH7.8) every day (each coverslip was washed twice with $2 \mathrm{ml}$ of sterilized PBS). Two milliliters of pentanediol was used to fix, after $20 \mathrm{~min}$, then the same way to deal with pentanediol. Crystal violet $(0.5 \%, 2 \mathrm{ml})$ was added and incubated at room temperature for $15 \mathrm{~min}$. Coverslips rinsed with distilled water until there was no purple liquid. To quantitate, $2 \mathrm{ml}$ of $95 \%$ (v/v) ethanol was added in each well to re-dissolve the crystal violet that closely combined with the coverslip. Finally, $200 \mu \mathrm{L}$ of solution was transferred to 96-well plates and the absorbance was read at $570 \mathrm{~nm}[12]$.

\subsection{Statistical Analysis}

All experiments were performed in triplicate and the data obtained from the experiments were presented as mean values with or without standard deviation and the differences between control and test were analyzed using Student's t-test

\section{Results}

\subsection{MIC of Oridonin against $P$. aeruginosa PAO1}

The influence of oridonin on PAO1 growth was examined by MTT method. This assay was aimed to rule out any antibacterial properties of oridonin that may inhibit growth of PAO1. The results showed that oridonin inhibited PAO1 growth at the concentration of $250 \mu \mathrm{g} / \mathrm{ml}$ (Table 1), while there was little effect on the growth of bacteria below $125 \mu$ $\mathrm{g} / \mathrm{ml}$. The MIC of oridonin against PAO1 was $125 \mu \mathrm{g} / \mathrm{ml}$. 
Table 1. MIC of Oridonin to Pseudomonas Aeruginosa (mean \pm SD)

\begin{tabular}{lllllllll}
\hline \multicolumn{1}{c}{ Oridonin $(\mu \mathrm{g} / \mathrm{ml})$} & 2000 & 1000 & 500 & 250 & 125 & 62.5 & 31.3 & 15.6 \\
Bacterial & 0.22 & 0.31 & 0.48 & 0.92 & 1.22 & 1.23 & 1.23 & 1.22 \\
Concentration $^{\mathrm{a}}$ & $\pm 0.02^{*}$ & $\pm 0.02 *$ & $\pm 0.03 *$ & $\pm 0.02^{*}$ & $\pm 0.04 * *$ & $\pm 0.04 * *$ & $\pm 0.03 * *$ & $\pm 0.03 * *$ \\
\hline
\end{tabular}

${ }^{a}$ Concentrations were expressed as the absorbance at $600 \mathrm{~nm}$, Data represent mean and standard deviation of three independent experiments. $* \mathrm{p} \leqslant 0.05, * * \mathrm{p} \leqslant 0.01$

\subsection{Effects of oridonin on QS-Regulated virulence factors in PAO1}

Table 2 shows the dose-dependent effect of oridonin on virulence factors in PAO1. Oridonin reduced variously pyocyanin production in PAO1 at different concentration in a dose-dependent manner, with $58.4 \%$ (MIC), $39.4 \%$ $(1 / 2 \mathrm{MIC})$ and $27.7 \%$ (1/4 MIC) reductions, respectively. The result shows oridonin reduced rhamnolipids production by $32.0 \%, 60.5 \%$ and $64.3 \%$ at MIC, $1 / 2 \mathrm{MIC}$, 1/4 MIC, respectively

A significant concentration-dependent decrease (25.9$40.9 \%$ ) of protease production was observed in the culture supernatant of PAO1 compared to control. Oridonin

reduced protease activity by $25.9 \%, 30.0 \%$ and $40.9 \%$ at MIC, 1/2 MIC, and 1/4 MIC, respectively (Table 2).

Elastase activity decreased substantially at subinhibitory concentrations $(31.3-125 \mu \mathrm{g} / \mathrm{ml})$ of oridonin with $42.4-58.6 \%$ reduction compared to the control. The results showed that there was significant difference between the experimental groups and the negative group ( $\mathrm{P}$ $<0.05)$.

\subsection{Effect on Biofilm Formation}

Sub-MICs were tested for biofilm inhibition in PAO1 using crystal violet assay. Sub-MICs of oridonin decrease biofilm Formation of PAO1. Biofilm formation was significantly inhibited by $40.3 \%, 48.4 \%$, and $57.7 \%$ at 31.3 , 62.5 , and $125 \mu \mathrm{g} / \mathrm{ml}$ concentrations, respectively.

Table 2. QSI Activity of Oridonin in Pseudomonas Aeruginosa (mean \pm SD)

\begin{tabular}{clllll}
\hline Oridonin $(\mu \mathrm{g} / \mathrm{ml})$ & \multicolumn{1}{c}{ Pyocyanine $^{\mathrm{a}}\left(\mathrm{IR}^{\mathrm{b}}\right)$} & \multicolumn{1}{c}{ Rhamnolipids $^{\mathrm{c}}\left(\mathrm{IR}^{\mathrm{b}}\right)$} & \multicolumn{1}{c}{ Protease $^{\mathrm{d}}\left(\mathrm{IR}^{\mathrm{b}}\right)$} & \multicolumn{1}{c}{ Elastase $^{\mathrm{e}}\left(\mathrm{IR}^{\mathrm{b}}\right)$} & \multicolumn{1}{c}{$\operatorname{Biofilms}^{\mathrm{f}}\left(\mathrm{IR}^{\mathrm{b}}\right)$} \\
\hline Control & $0.137 \pm 0.025$ & $0.824 \pm 0.032$ & $0.220 \pm 0.019$ & $0.494 \pm 0.027$ & $0.397 \pm 0.040$ \\
31.3 & $0.099 \pm 0.022(27.7)$ & $0.560 \pm 0.024(32.0)$ & $0.163 \pm 0.015(25.9)$ & $0.284 \pm 0.022(42.4)^{*}$ & $0.238 \pm 0.033(40.3)^{*}$ \\
62.5 & $0.084 \pm 0.023(39.4)^{*}$ & $0.325 \pm 0.018(60.7)^{*}$ & $0.155 \pm 0.012(30.0)^{*}$ & $0.246 \pm 0.021(50.3)^{*}$ & $0.206 \pm 0.027(48.4)^{*}$ \\
125 & $0.058 \pm 0.017(58.4)^{* *}$ & $0.294 \pm 0.017(64.3)^{* *}$ & $0.113 \pm 0.012(40.9)^{*}$ & $0.204 \pm 0.018(58.6)^{* *}$ & $0.169 \pm 0.016(57.7)^{* *}$
\end{tabular}

a Pyocyanin concentrations were expressed as the absorbance at 520 $\mathrm{nm}^{\mathrm{b}}{ }^{\mathrm{b}}$ Inhibiting Rate. ${ }^{\mathrm{c}}$ Rhamnolipids concentrations were expressed as the absorbance at $421 \mathrm{~nm}$. ${ }^{\mathrm{d}}$ Protease activity is expressed as the absorbance at $440 \mathrm{~nm}$. ${ }^{\circ}$ Elastase activity is expressed as the absorbance at $495 \mathrm{~nm}$. ${ }^{\text {B Biofilm formation is }}$ expressed at $470 \mathrm{~nm}$ after incubation with crystal violet. Data represent mean and standard deviation of three independent experiments. Values in parentheses indicate percent reduction over control. $* \mathrm{p} \leq 0.05, * * \mathrm{p} \leq 0.01$

\section{Discussion}

Pseudomonas aeruginosa is one of the pathogens that can escape the treatment of various antibiotics. It is widely distributed in nature, normal skin, intestinal and respiratory tract, which is the main cause of respiratory tract infection, urinary tract infection, septicemia, osteomyelitis and skin infections[13]. QS enables the $P$. aeruginosa to synthesize and release a large numbers of extracellular virulence factors, such as pyocyanin, rhamnolipids, proteases, etc. Some research has found that an obvious reduction was detected in the secretion of virulence factors, biofilm formation, invasiveness of infected hosts when QS system was blocked[14] .

Pyocyanin can be easily found in the sputum of cystic fibrosis (CF) patients and it can cause detrimental effects toward lung epithelial cells[15]. Pyocyanin formation is regulated by a complex synchrony of rhlR-rhlI and lasRlasI whereby interferences in these systems led to the deficiency of pyocyanin formation[16]. Our study shows pyocyanin production has a steady decrease with the increasing concentrations of oridonin.

Rhamnolipids are vital microbial derived surfactants produced by $P$. aeruginosa and under the control of Las and Rhl systems. They are important to bacteria motility. The rhlA mutant which lacks rhamnolipids production completely loses its swarming ability. Furthermore, many studies showed that QS positively regulates rhamnolipids production. Rhamnolipids are made up of two fatty acid molecules and rhamnose residues, which is controlled by Rhl system, mainly by rhlAB synthetic gene cluster expression[17]. It can decrease surface tension and biofilm development. Our result shows oridonin reduced rhamnolipids production by $32.0-64.3 \%$ without interfering the growth of PAO1. Similar reduction in pyocyanin production was recorded in literature[18].

The hydrolytic enzymes such as protease and elastase are known to affect the host cell proteins in the infected tissues and facilitate bacterial invasion and growth. LasI/LasR system regulates the genes responsible for lasA(protease) [19]. In the present study, preincubation with oridonin created dose-dependent inhibition of elastase 
and protease production. These data corroborated with the literature where, elastase activity and proteolytic activity of $P$. aeruginosa was decreased by plant extracts and essential oils [20]

Quorum sensing are known to play significant role in biofilm formation. P. aeruginosa biofilm can effectively resist the attack of antibiotics and immune system. We found that oridonin at Sub-MICs inhibited the biofilm biomass by $40.3-57.7 \%$ significantly $(P \leqslant 0.05)$ with no significant growth inhibition on PAO1.

Based on our data and available literature, it could be assumed that oridonin might have inhibited the QSsystems in $P$. aeruginosa, as a potential candidate for exploiting as anti-infective agent in modern phytomedicine.

\section{References}

1. Andersson, D. I. (2003) Persistence of antibiotic resistant bacteria, Curr Opin Microbiol. 6, 452-6.

2. Walsh, C. (2003) Where will new antibiotics come from?, Nat Rev Microbiol. 1, 65-70.

3. Reading, N. C. \& Sperandio, V. (2006) Quorum sensing: the many languages of bacteria, FEMS Microbiol Lett. 254, 1-11.

4. Mellbye, B. \& Schuster, M. (2011) The sociomicrobiology of antivirulence drug resistance: a proof of concept, mBio. 2.

5. Managò, A., Becker, K. A., Carpinteiro, A., Wilker, B., Soddemann, M., Seitz, A. P., Edwards, M. J., Grassmé, H., Szabò, I. \& Gulbins, E. (2015) Pseudomonas aeruginosa pyocyanin induces neutrophil death via mitochondrial reactive oxygen species and mitochondrial acid sphingomyelinase, Antioxid Redox Signal. 22, 1097-1110.

6. Alhede, M., Bjarnsholt, T., Givskov, M. \& Alhede, M. (2014) Pseudomonas aeruginosa biofilms: mechanisms of immune evasion, Adv Appl Microbiol. 86, 1-40.

7. Chudnicka A, M. G. (2005) Research of enzymatic activities of fresh juice and water infusions from dry herbs, Journal of EthnoPharmacology. 99, 281-287.

8. Siraj, D., Debanjan, J. \& Raj, M. (2016) Piper betleleaf extract affects the quorum sensing and hence virulence of Pseudomonas aeruginosa PAO1, Biotech. 6, 18-20.

9. Rasamiravaka, T., Vandeputte, O. \& Pottier, L. (2015) Pseudomonas aeruginosa Biofilm Formation and Persistence, along with the Production of Quorum Sensing-Dependent Virulence Factors, Are Disrupted by a Triterpenoid Coumarate Ester Isolated from Dalbergia trichocarpa, a Tropical Legume, Plos One. 10, e0132791.

10. Husain, F., Ahmad, I. \& Al-Thubiani, A. (2017) Leaf Extracts of Mangifera indica L. Inhibit Quorum
Sensing - Regulated Production of Virulence Factors and Biofilm in Test Bacteria, Front Microbiol, 827830.

11. Rathinam, P., Vijay Kumar, H. \& Viswanathan, P. (2017) Eugenol exhibits anti-virulence properties by competitively binding to quorum sensing receptors, Biofouling. 33, 624-639.

12. Busetti, A., Shaw, G. \& Megaw, J. (2014) Marinederived quorum-sensing inhibitory activities enhance the antibacterial efficacy of tobramycin against Pseudomonas aeruginosa, Marine Drugs. 13, 1-28.

13. Doshi, H., Chua, K. \& Kagda, F. (2011) Multidrug resistant pseudomonas infection in open fractures post definitive fixation leading to limb loss: A report of three cases, International Journal of Case Reports \& Images. 2, 1-6.

14. Lee, J. \& Zhang, L. (2015) The hierarchy quorum sensing network in Pseudomonas aeruginosa, Protein and Cell. 6, 26-41.

15. Usher, L., Lawson, R. \& Geary, I. (2002) Induction of neutrophil apoptosis by the Pseudomonas aeruginosa exotoxin pyocyanin: a potential mechanism of persistent infection, Journal of Immunology. 168, 1861-8.

16. Cao, H., Krishnan, G. \& Goumnerov, B. (2001) A quorum sensing-associated virulence gene of Pseudomonas aeruginosa encodes a LysR-like transcription regulator with a unique self-regulatory mechanism, Proc Natl Acad Sci USA. 98, 14613-8.

17. Bjarnsholt, T., Jensen, P. O., Burmolle, M., Hentzer, M., Haagensen, J. A. J., Hougen, H. P., Calum, H., Madsen, K. G., Moser, C., Molin, S., Hoiby, N. \& Givskov, M. (2005) Pseudomonas aeruginosa tolerance to tobramycin, hydrogen peroxide and polymorphonuclear leukocytes is quorum-sensing dependent, Microbiology (Reading). 151, 373-383.

18. Kim, H. S., Lee, S. H., Byun, Y. \& Park, H. D. (2015) 6-Gingerol reduces Pseudomonas aeruginosa biofilm formation and virulence via quorum sensing inhibition, Sci Rep. 5, 8656.

19. Adonizio, A., Kong, K. F. \& Mathee, K. (2008) Inhibition of quorum sensing-controlled virulence factor production in Pseudomonas aeruginosa by South Florida plant extracts, Antimicrob Agents Chemother. 52, 198-203.

20. Husain, F. M. \& Ahmad, I. (2013) Quorum sensing inhibitors from natural products as potential novel antiinfective agents, Drug Future. 38, 691-706. 